\title{
REGULARITIES OF LINE STRENGTHS IN SPECTRA OF NI, FI, AND NeII
}

\author{
J. MuSIELOK \\ Institute of Physics, Opole University, Oleska 48, 45-052 Opole, Poland
}

(Received April 21, 1998; in final form June 1, 1998)

\begin{abstract}
Recently experimentally determined individual (fine structure) line strengths for the spectra of NI, FI, and NeII are analysed, searching for regularities resulting from similarities in the structure of these species. Strengths of spectral lines of the prominent $3 s-3 p$ and $3 p-3 d$ transition arrays are investigated. The absolute scales of these experimentally derived data are based on independently determined lifetimes for excited levels of NI, FI, and NeII. The simple Coulomb approximation method was applied for calculations of the matrix elements for the "jumping" electron in order to obtain an average trend in line strengths of the studied emitters. In the case of the isoelectronic species (FI, NeII), additionally the trend resulting from the perturbation theory is taken for comparison. Except the trend study of the absolute scale, the strengths of individual lines within analogous multiplets (in a relative scale) are investigated and compared with data resulting from LS coupling scheme and with semi-empirical calculations.
\end{abstract}

PACS numbers: $32.30 .-r, 32.70$. Cs

\section{Introduction}

In recent years a large number of transition strengths for light elements has been calculated within the international opacity project $[1,2]$. These calculations provide data characterising the total strengths of multiplets. Other advanced calculations [3-8], pursued down to the fine structure level, are restricted only to a few light species and a significant lesser number of transitions. In a recent sequence of papers the relativistic quantum defect orbital (RQDO) method has been applied for determination of oscillator strengths of light atoms with two or more valence electrons, and the LS coupling scheme was found to be a useful approximation [9-11]. Also recently a few experiments have been performed, applying wall-stabilised arcs [12-16] and hollow cathode discharges [17], yielding accurate relative and/or absolute transition probabilities for light elements.

In the three analysed species: NI, FI, and NeII some regularities in their optical spectra are expected arising from similar (NI, FI) or identical (FI, NeII) 
electron configuration of the emitter's core. The cores $1 s^{2} 2 s^{2} 2 p^{2}$ (NI) and $1 s^{2} 2 s^{2} 2 p^{4}$ (FI, NeII) lead obviously to identical parent terms and consequently to terms of the same multiplicity. For the prominent transition arrays with a fixed principal quantum number $n=3(3 s-3 p$ and $3 p-3 d)$ a strong electrostatic interaction (LS coupling) between the "jumping" electron and the emitter's core has to be expected. Thus, relative line strengths within analogous multiplets should be exactly the same [18] or at leasi, as stated in Ref. [11], should be reasonably well described by this model.

However, recent measurements reveal that noticeable departures from LS coupling scheme occur for CI [15], NI [12], NII [13], OII [14], FI [16], and NeII [17]. Therefore studies of regularities within analogous transitions in species of similar or identical electron configurations seem to be useful and important.

The accuracy of relative line strengths measurements within multiplets prevails obviously the accuracy of determination of the absolute scale. In the analysed data sets of NI, FI, and NeII the absolute scales of line strengths were established on hand of a few lifetimes of excited levels belonging to the $3 p$ or $3 d$ electron configuration for NI, FI, and NeII. Usually the uncertainty of the lifetime determination yields the major contribution to the uncertainty of absolute line strengths. For details see Refs. $[12 ; 16,17]$.

For that reason the present study is divided into two parts: (a) the comparison of relative line strengths within multiplets determined in the papers $[12,16,17]$, and (b) the comparison of averaged absolute scales based on the respective lifetimes with (i) the trend resulting from the simple Coulomb approximation (CA) method [19], and in the case of the isoelectronic species FI, NeII additionally with (ii) the scaling resulting from the perturbation theory [20, 21].

The perturbation theory yields the result that the $f$ value (oscillator strength) for a given transition along an isoelectronic sequence depends on the nuclear charge of the emitter as

$$
f=f_{0}+f_{1} Z^{-1}+f_{2} Z^{-2}+\ldots
$$

where $Z$ is the nuclear charge of the emitter and the constant term $f_{0}$ is a hydrogenic $f$ value. For our studied transition arrays $(3 s-3 p$ and $3 p-3 d)$ the above formula is even more simple, because for spectral lines with $\Delta n=0$, the hydrogenic term $f_{0}$ obviously vanishes.

The trend in absolute line strengths resulting from the CA method can be easily calculated from the corresponding matrix elements, knowing the energy levels of the studied transition and the respective ionization energy of the emitter.

\section{Data reduction and results}

In the papers $[12,16,17]$ the quantum transitions of the spectra of NI, FI and NeII are characterised by quoting the respective transition probabilities (Einstein coefficients). In order to search for regularities in spectra the transition probabilities were converted into the corresponding line strengths (in atomic units), applying the following relation [21]:

$$
S_{i k}=4.9356 \times 10^{-19} g_{k} \lambda_{k i}^{3} A_{k i}
$$

where $g_{k}$ is the statistical weight of the upper level of the transition, $\lambda_{k i}$ is the wavelength in angstrom units, $A_{k i}$ is the transition probability in $\mathrm{s}^{-1}$. 
As mentioned above, the emission spectroscopy method yields rather accurate relative transition probabilities or relative line strengths. The absolute values of these quantities are usually charged with significantly larger errors, mainly originating from uncertainties of the lifetime values applied in the normalization procedure. In the analysed papers $[12,16,17]$ the determination accuracies of.relative line strengths within multiplets are typically about $2-3 \%$ for the strong lines and not exceeding $10 \%$ for the weak ones.

\subsection{Comparison of relative line strengths}

In Table I results for 6 analogous multiplets of NI, FI, and NeII, for which complete sets of fine structure lines have been determined respectively in Refs. $[12,16,17]$, are presented. The line strengths within each multiplet are normalised to a sum equal to 100 . The experimental data are compared with those resulting from the LS coupling scheme and with the semi-empirical results of $\mathrm{Ku}$ rucz and Peytremann [22], because this comprehensive data set contains results for all three studied emitters.

The results for all analysed $3 s-3 p$ multiplets of NI show rather good agreement with the LS coupling data. Remarkable departures are encountered for two very weak lines only. The analogous line strengths for the two heavier isoelectronic species, FI and NeII, adhere well to each other but differ significantly from LS prediction. On the other hand, the consistence with the semi-empirical data of Kurucz and Peytremann is surprisingly good and worth stressing. Particularly, the strengths of the weaker lines agree much better with experimental data than with those resulting from the LS coupling scheme.

In Table II data for analogous NI and FI lines belonging to the $3 p-3 d$ transition array are compiled. Since for majority of these multiplets the experimental sets are not complete, the line strengths were normalised always to the line, which according to the LS coupling scheme is the strongest one. Again data taken from Ref. [22] are listed for comparison.

As can be seen the discrepancies between the NI and FI line strengths are very large with only one exception - the results for the ${ }^{2} S^{\circ}-{ }^{2} P$ multiplet, where the consistence with LS data is rather good. Quite reasonable agreement between the experimental results for NI and the LS data is found for the multiplet ${ }^{4} D^{\circ}-{ }^{4} F$, while the respective data for FI reveal significant discrepancies. In the case of the multiplet ${ }^{4} P^{\circ}-{ }^{4} D$ the LS coupling fails noticeably even for the NI spectrum, exhibiting large departures for weak components. For the multiplets ${ }^{4} D^{\circ}-{ }^{4} P$ and ${ }^{4} P^{\circ}{ }^{4} P$ the discrepancies between the results for NI and FI, and the departures from data resulting from LS coupling scheme are immense, exceeding sometimes (in the case of FI) a factor of 1000 . The agreement between experimental results and the semi-empirical data is again much better - the largest discrepancy reaches a factor of 4 only (transition ${ }^{4} D_{3 / 2}^{\circ}-{ }^{4} P_{3 / 2}$ ). In general the semi-empirical $g f$ values, listed in Ref. [22], allow us to predict at least the correct schedule in the strengths of components within each multiplet. 
TABLE I

Comparison of experimentally determined relative line strengths of the $3 s-3 p$ transition array for the spectra of NI [12], FI [16], and NeII [17] with the data obtained from the LS coupling scheme and with the semi-empirical results of Kurucz and Peytremann [22]. The line strengths within each multiplet are normalised to a sum of 100 .

\begin{tabular}{|c|c|c|c|c|c|c|c|c|}
\hline \multirow{2}{*}{$3 s-3 p$} & \multirow[b]{2}{*}{$g_{i}-g_{k}$} & \multirow[b]{2}{*}{$\mathrm{LS}$} & \multicolumn{2}{|c|}{$\mathrm{NI}$} & \multicolumn{2}{|c|}{ FI } & \multicolumn{2}{|c|}{ NeII } \\
\hline & & & [12] & {$[22]$} & [16] & {$[22]$} & [17] & {$[22]$} \\
\hline \multirow[t]{3}{*}{${ }^{4} P-{ }^{4} S^{\circ}$} & $6-4$ & 50.0 & 51.7 & 50.3 & 42.5 & 42.1 & 43.6 & 46.9 \\
\hline & $4-4$ & 33.3 & 33.1 & 33.1 & 35.8 & 35.7 & 36.0 & 34.5 \\
\hline & $2-4$ & 16.7 & 15.0 & 16.6 & 21.7 & 22.2 & 20.4 & 18.6 \\
\hline \multirow[t]{7}{*}{${ }^{4} P-{ }^{4} P^{0}$} & $6-6$ & 35.0 & 36.4 & 35.0 & 39.3 & 40.2 & 39.3 & 37.2 \\
\hline & $4-4$ & 4.4 & 5.5 & 4.4 & 5.0 & 5.2 & 4.5 & 4.8 \\
\hline & $2-2$ & 2.8 & 2.7 & 2.8 & 2.5 & 2.1 & 2.5 & 2.5 \\
\hline & $6-4$ & 15.0 & 14.9 & 15.0 & 17.5 & 18.2 & 16.2 & 16.5 \\
\hline & $4-2$ & 13.9 & 14.5 & 14.0 & 14.7 & 14.7 & 15.5 & 14.2 \\
\hline & $4-6$ & 15.0 & 12.8 & 14.9 & 10.5 & 9.9 & 11.9 & 12.6 \\
\hline & $2-4$ & 13.9 & 13.2 & 13.9 & 10.5 & 9.7 & 10.1 & 12.1 \\
\hline \multirow[t]{8}{*}{${ }^{4} P-{ }^{4} D^{\circ}$} & $6-8$ & 39.9 & 39.1 & 40.5 & 42.0 & 40.1 & & \\
\hline & $4-6$ & 21.0 & 22.2 & 21.2 & 23.2 & 23.8 & & \\
\hline & $2-4$ & 8.35 & 9.2 & 8.3 & 9.1 & 9.9 & & \\
\hline & $6-6$ & 9.0 & 8.0 & 9.1 & 6.0 & 5.8 & & \\
\hline & $4-4$ & 10.7 & 10.5 & 10.0 & 9.3 & 9.8 & & \\
\hline & $2-2$ & 8.35 & 8.8 & 8.3 & 8.7 & 9.0 & & \\
\hline & $6-4$ & 1.0 & 0.7 & 1.0 & 0.5 & 0.4 & & \\
\hline & $4-2$ & 1.7 & 1.5 & 1.6 & 1.2 & 1.2 & & \\
\hline \multirow[t]{2}{*}{${ }^{2} P-{ }^{2} S^{\circ}$} & $4-2$ & 66.7 & & & 88.4 & 89.9 & 85.5 & 86.6 \\
\hline & $2-2$ & 33.3 & & & 11.6 & 10.1 & 14.5 & 13.4 \\
\hline \multirow[t]{4}{*}{${ }^{2} P-{ }^{2} P^{0}$} & $4-4$ & 55.6 & 56.7 & 55.1 & 48.3 & 47.8 & 49.3 & 52.1 \\
\hline & $2-2$ & 22.2 & 22.0 & 22.4 & 29.8 & 30.5 & 31.0 & 29.8 \\
\hline & $4-2$ & 11.1 & 11.0 & 11.3 & 3.7 & 3.1 & 4.3 & 3.8 \\
\hline & $2-4$ & 11.1 & 10.2 & 11.2 & 18.2 & 18.6 & 15.4 & 14.3 \\
\hline \multirow[t]{3}{*}{${ }^{2} P-{ }^{2} D^{\circ}$} & $4-6$ & 60.0 & 59.7 & 60.3 & 58.7 & 59.1 & 57.9 & 60.1 \\
\hline & $2-4$ & 33.3 & 34.1 & 33.1 & 31.1 & 29.8 & 32.1 & 31.6 \\
\hline & $4-4$ & 6.7 & 6.2 & 6.6 & 10.2 & 11.1 & 10.0 & 8.3 \\
\hline
\end{tabular}


TABLE II

Comparison of experimentally determined relative line strengths within multiplets belonging to the $3 p-3 d$ transition array in the spectra of NI [12] and FI [16], with those predicted by the LS coupling and the semi-empirical data of Kurucz and Peytremann [22]. The line strengths within each multiplet are normalised always to the strongest line predicted by the LS coupling scheme.

\begin{tabular}{|c|c|c|c|c|c|c|}
\hline \multirow{2}{*}{$3 p-3 d$} & \multirow[b]{2}{*}{$g_{i}-g_{k}$} & \multirow[b]{2}{*}{ LS } & \multicolumn{2}{|c|}{ NI } & \multicolumn{2}{|c|}{ FI } \\
\hline & & & {$[12]$} & [22] & [16] & {$[22]$} \\
\hline \multirow[t]{7}{*}{${ }^{4} D^{\circ}-{ }^{4} F$} & $8-10$ & 100 & 100 & 100 & 100 & 100 \\
\hline & $6-8$ & 68.6 & 68.0 & 69.2 & 34.2 & 38.6 \\
\hline & $4-6$ & 45.0 & 45.5 & 45.7 & 28.4 & 32.4 \\
\hline & $2-4$ & 28.0 & 27.9 & 21.4 & 17.1 & 20.0 \\
\hline & $8-8$ & 11.4 & 8.6 & 6.8 & 0.89 & 1.0 \\
\hline & $6-6$ & 14.6 & 11.8 & 10.3 & 5.1 & 4.0 \\
\hline & $4-4$ & 11.2 & 10.6 & 8.1 & 6.2 & 7.7 \\
\hline \multirow[t]{4}{*}{${ }^{4} D^{\circ}-{ }^{4} D$} & $8-8$ & 100 & 100 & 100 & 100 & 100 \\
\hline & $6-6$ & 50.3 & 40.2 & 56.0 & 56.9 & 46.3 \\
\hline & $4-4$ & 23.3 & 16.6 & 22.8 & 19.8 & 17.4 \\
\hline & $8-6$ & 16.6 & 22.0 & 22.4 & 14.9 & 15.8 \\
\hline \multirow[t]{5}{*}{${ }^{4} D^{\circ}-{ }^{4} P$} & $8-6$ & 100 & 100 & 100 & 100 & 100 \\
\hline & $6-6$ & 22.5 & 162 & 673 & 3380 & 7540 \\
\hline & $4-4$ & 26.6 & 168 & 367 & 469 & 1900 \\
\hline & $4-6$ & 2.5 & 53.3 & 14.5 & 3910 & 6670 \\
\hline & $2-4$ & 4.2 & 34.8 & 43.5 & 1100 & 2090 \\
\hline \multirow[t]{8}{*}{${ }^{4} P^{\circ}-{ }^{4} D$} & $6-8$ & 100 & 100 & 100 & 100 & 100 \\
\hline & $4-6$ & 52.6 & 38.6 & 43.5 & 32.4 & 37.4 \\
\hline & $2-4$ & 20.9 & 12.9 & 13.3 & 8.4 & 9.5 \\
\hline & $6-6$ & 22.5 & 36.1 & 39.7 & 29.0 & 38.9 \\
\hline & $4-4$ & 26.8 & 32.1 & 34.7 & 26.1 & 30.9 \\
\hline & $2-2$ & 20.9 & 18.7 & 20.0 & 13.1 & 15.3 \\
\hline & $6-4$ & 2.5 & 6.8 & 7.2 & 5.5 & 7.0 \\
\hline & $4-2$ & 4.3 & 7.6 & 8.3 & 7.1 & 12.1 \\
\hline \multirow[t]{6}{*}{${ }^{4} P^{\circ}-{ }^{4} P$} & $6-6$ & 100 & 100 & 100 & 100 & 100 \\
\hline & $2-2$ & 7.9 & 45.9 & 33.5 & 35.0 & 54.5 \\
\hline & $6-4$ & 42.8 & 63.7 & 53.5 & 55.4 & 63.6 \\
\hline & $4-2$ & 39.5 & 54.6 & 49.6 & 133 & 139 \\
\hline & $4-6$ & 42.8 & 174 & 154 & 95.2 & 116 \\
\hline & $2-4$ & 39.5 & 152 & 122 & 284 & 302 \\
\hline \multirow[t]{2}{*}{${ }^{2} S^{\circ}-{ }^{2} P$} & $2-4$ & 100 & 100 & 100 & 100 & 100 \\
\hline & $2-2$ & 50.0 & 51.7 & 65.5 & 57.5 & 185 \\
\hline
\end{tabular}




\subsubsection{Comparison of absolute line strengths for $3 s-3 p$ transitions of FI and NeII}

In the paper [16] transition probabilities for 40 spectral lines of FI, belonging to the $3 s-3 p$ transition array have been determined in an absolute scale. For the next member of the isoelectronic sequence (NeII), the determination in an absolute scale was possible for 30 lines [17]. A direct comparison of absolute line strengths of these two data sets was possible only for 25 analogous transitions, among them for 5 intersystem lines.

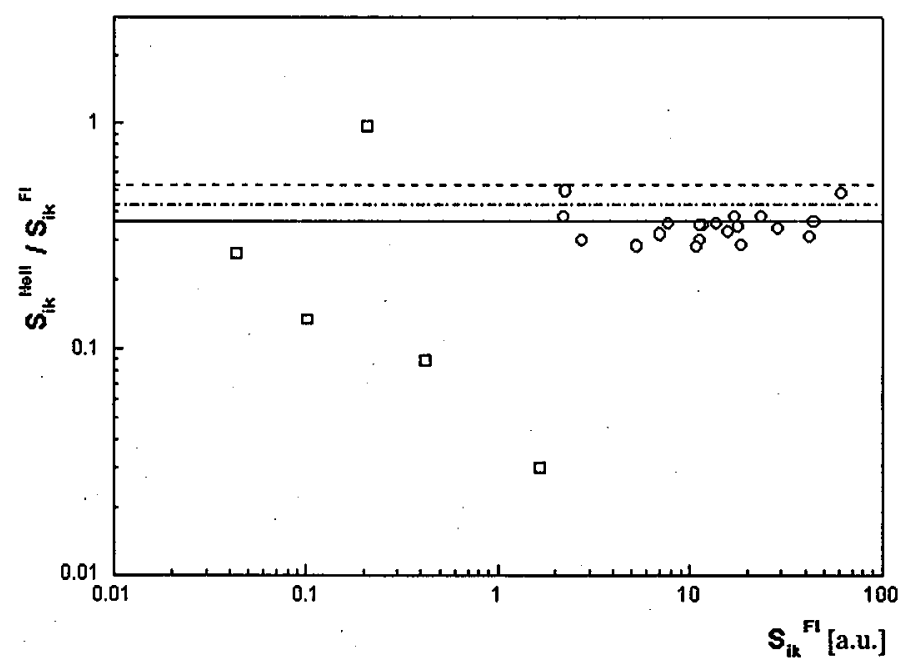

Fig. 1. Line strength ratios of analogous transitions of NeII and FI versus the fluorine line strengths. Solid line - weighted mean ratio, dashed-dotted line - perturbation theory, dashed line - Coulomb approximation method. Circles represent LS - allowed, while squares LS - forbidden transitions.

In Fig. 1 the ratios of analogous line strengths, $S_{i k}^{\mathrm{NeII}} / S_{i k}^{\mathrm{FI}}$, are plotted as a function of FI line strengths (in atomic units). In Fig. 2 the respective wavelength ratios are plotted versus the wavelength of the pertinent FI transitions. The circles represent the data for the LS allowed transitions, while the squares for the 5 intersystem lines. If the data for the intersystem lines are omitted and the individual strength ratios are weighted with the corresponding strengths of the FI line, a mean $S_{i k}^{\mathrm{NeII}} / S_{i k}^{\mathrm{FI}}$ value of 0.36 is obtained (solid line in Fig. 1). Using the data of Ref. [22] a mean ratio of 0.44 is evaluated.

As can be seen from Fig. 2, the wavelength ratio $\left(\lambda^{\mathrm{NeII}} / \lambda^{\mathrm{FI}}\right)$ varies from 0.44 to 0.51 . The FI lines appear in the red part of the spectrum, while the corresponding transitions in NeII are in the UV range. For the mean wavelength ratio one obtains: $\left(\lambda^{\mathrm{NeII}} / \lambda^{\mathrm{FI}}\right)=0.48$, the inclusion or omission of the intersystem lines does not influence significantly the final result.

Applying formula (1) with $Z=9$ and $Z=10$ for FI and NeII, respectively, and restricting it to the terms with $Z^{-1}$, one obtains a mean oscillator strength ratio $\left(f_{i k}^{\mathrm{NeII}} / f_{i k}^{\mathrm{FI}}\right)$ of about 0.90 . In order to convert the oscillator strength ratio into 


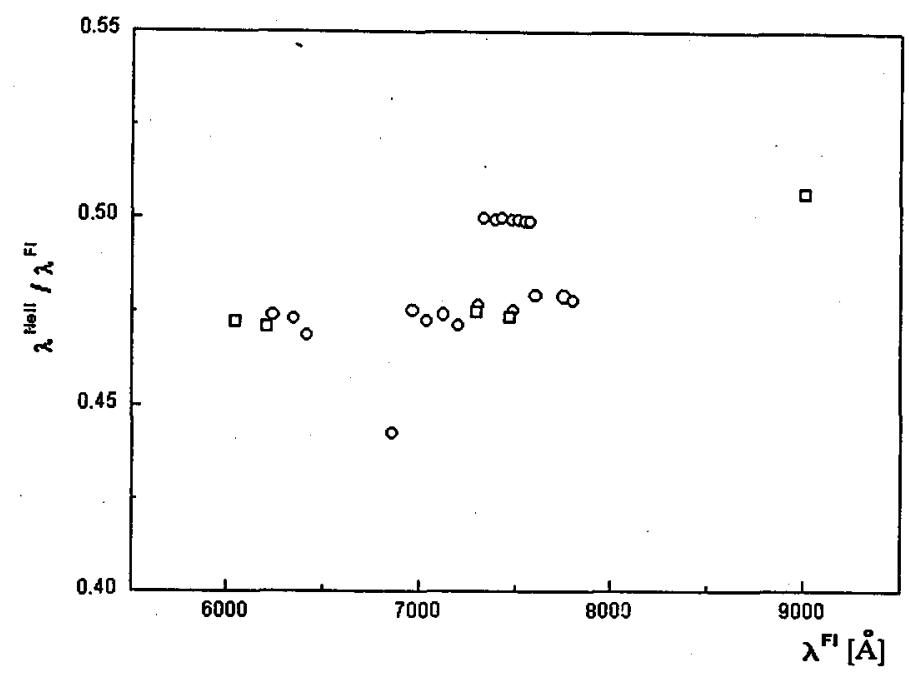

Fig. 2. Wavelength ratios of analogous transitions of NeII and FI versus the fluorine wa velengths. Circles represent LS - allowed, while squares LS - forbidden transitions.

the line strength ratio, the corresponding wavelength ratio $\left(\lambda^{\mathrm{NeII}} / \lambda^{\mathrm{FI}}\right)=0.48$, has to be taken into account. In this manner a mean line strength ratio $\left(S_{i k}^{\mathrm{NeII}} / S_{i k}^{\mathrm{FI}}\right)=$ 0.43 is obtained (dashed-dotted line in Fig. 1). This ratio agrees very well with the value obtained from the semi-empirical results [22] and is only about $20 \%$ larger than those resulting from the direct comparison of independently measured absolute line strengths. Since the lifetime values, which the absolute scales of the transition probability data $[16,17]$ are based on, have uncertainties of the same order, the agreement can be regarded as being satisfactory.

The mean line strength ratio $\left(S_{i k}^{\mathrm{NeII}} / S_{i k}^{\mathrm{FI}}\right)$ was also evaluated by applying the Coulomb approximation method. The effective principal quantum numbers were calculated from the corresponding excitation and ionization energies of FI and NeII, taken from Ref. [23]. In this manner, for the studied multiplets of the transition array $3 s-3 p$, a mean ratio of 0.53 was evaluated (dashed line in Fig. 1), which prevails those resulting from direct measurements by a factor of 1.47 , and those obtained from the perturbation theory by a factor of 1.23 .

\subsubsection{Comparison of absolute line strengths of $3 s-3 p$ transitions of NI and FI}

Among the analysed data sets of NI [12] and FI [16] only 25 results for analogous transitions are quoted, belonging to 5 multiplets. The mean ratio $\left(S_{i k}^{\mathrm{FI}} / S_{i k}^{\mathrm{NI}}\right)$, weighted with corresponding NI line strengths amounts 0.97 . The ratios for individual lines are ranging from 0.3 to 1.9. The largest scatter is observed for lines belonging to the multiplet ${ }^{2} P-{ }^{2} P^{\circ}$, the results for NI adhere well, while those for FI show large departures from LS coupling results. Using the same set of line strengths from Ref. [22], the corresponding ratio of 0.78 is evaluated. The mean ratio obtained from the $\mathrm{CA}$ is 0.80 and agree very well with the latter but is about $20 \%$ smaller than the "experimental" $(0.97)$ one. 


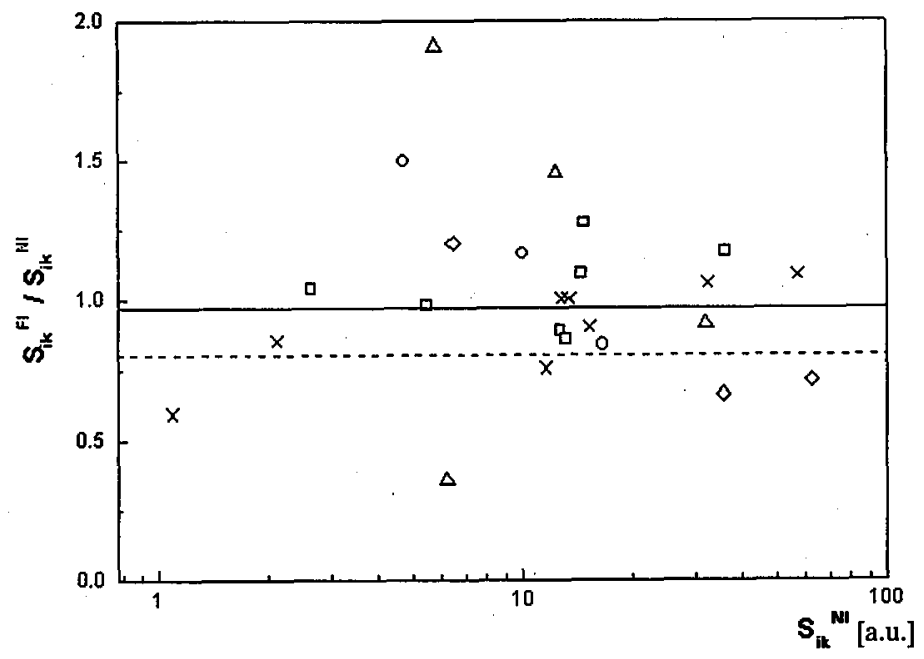

Fig. 3. Line strength ratios of analogous $3 s-3 p$ transitions of FI and NI as a function of the nitrogen line strengths. Solid line - weighted mean ratio, dashed line - ratio resulting from Coulomb approximation method. Various symbols represent different multiplets.

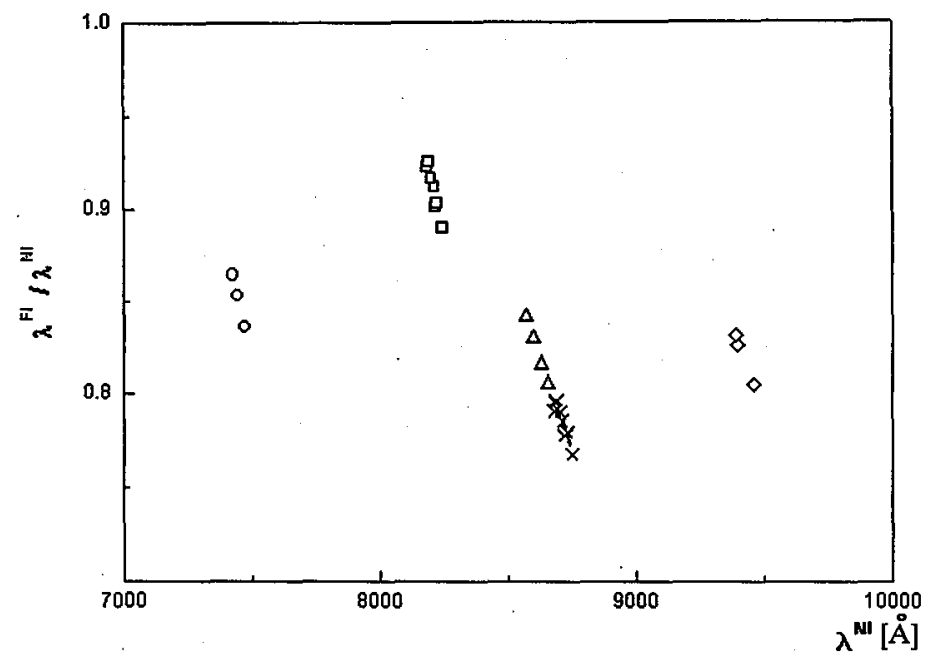

Fig. 4. Wavelength ratios of analogous $3 s-3 p$ transitions of FI and NI as a function of the nitrogen wavelengths. Various symbols represent different multiplets.

In Fig. 3 the individual ratios $S_{i k}^{\mathrm{FI}} / S_{i k}^{\mathrm{NI}}$ are plotted versus the NI line strengths. The solid line represents the weighted mean strength ratio, while the dotted line the ratio resulting from the $\mathrm{CA}$ method.

In Fig. 4 the respective wavelengths are compared. As can be seen, the wavelength ratio varies from 0.77 to 0.92 , and within each multiplet a clear trend 
is observed, indicating for a systematic departure of the energy structure of the elements from the LS coupling scheme (Lande interval rule).

\subsubsection{Comparison of absolute line strengths of $3 p-3 d$ transitions of NI and FI}

For the $3 p-3 d$ transition array 32 results for analogous spectral lines are listed in the analysed data sets $[12,16]$. In Fig. 5 the ratio $\left(S_{i k}^{\mathrm{FI}} / S_{i k}^{\mathrm{NI}}\right)$ is plotted versus the line strengths of the nitrogen data. The scatter of the data, compared with that of the $3 s-3 p$ transitions, is significantly larger ranging from about 0.04 to 3.0. Extreme large discrepancies are observed for lines belonging to the multiplet ${ }^{4} D^{\circ}-{ }^{4} P$. This irregularity can be explained in terms of a substantial departure from the LS coupling scheme in the case of NI and an entirely break-down in the case of FI. The spectral line, which according to the LS coupling should be the strongest within this multiplet, is the weakest one observed experimentally in the FI spectrum (see also Table II).

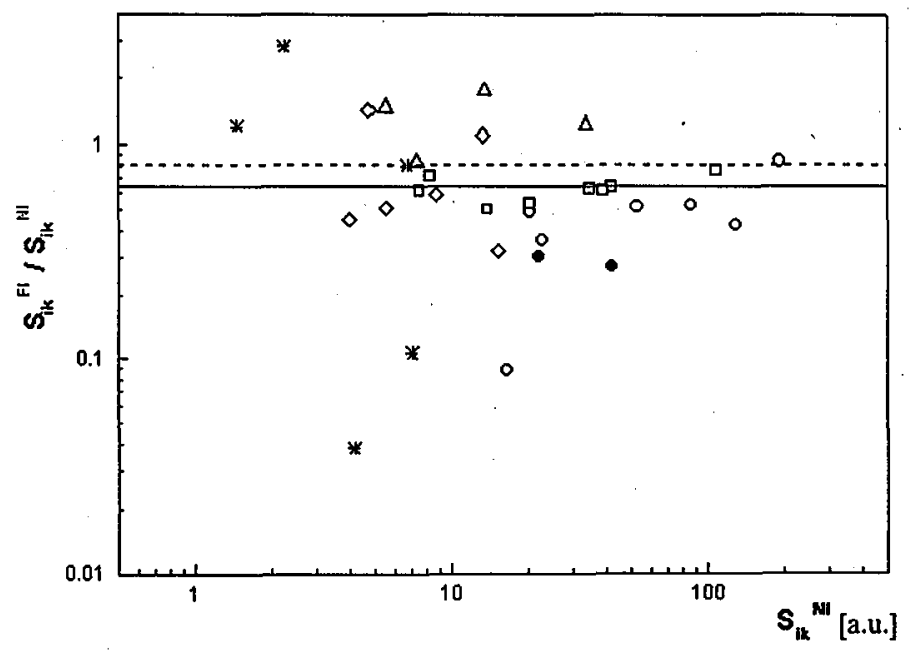

Fig. 5. The same as in Fig. 3 for $3 p-3 d$ transitions.

On the contrary the results for the two strongest multiplets: ${ }^{4} D^{\circ}-{ }^{4} F$ (open circles) and ${ }^{4} P^{\circ}-{ }^{4} D$ (squares) exhibit a very regular trend, with only one exception, caused evidently by the violation of the LS coupling scheme in the case of the FI spectrum, the line at $867.263 \mathrm{~nm}$ (transition ${ }^{4} D_{7 / 2}^{\circ}-{ }^{4} F_{7 / 2}$ ) is nearly one order of magnitude too weak if compared with the analogous line in the NI spectrum and with the LS prediction. It is worth stressing that this "irregularity" is correctly predicted by Kurucz and Peytremann [22] (see also Table II).

The mean experimental ratio, weighted with the NI line strengths $S_{i k}^{\mathrm{FI}} / S_{i k}^{\mathrm{NI}}$ amounts 0.65 (solid line), and agrees very well with the respective value $\left(S_{i k}^{\mathrm{FI}} / S_{i k}^{\mathrm{NI}}=\right.$ 0.67 ) obtained in the same way from the semi-empirical data set [22], while the CA yields 0.82 , which is again within the uncertainty of experiments. 


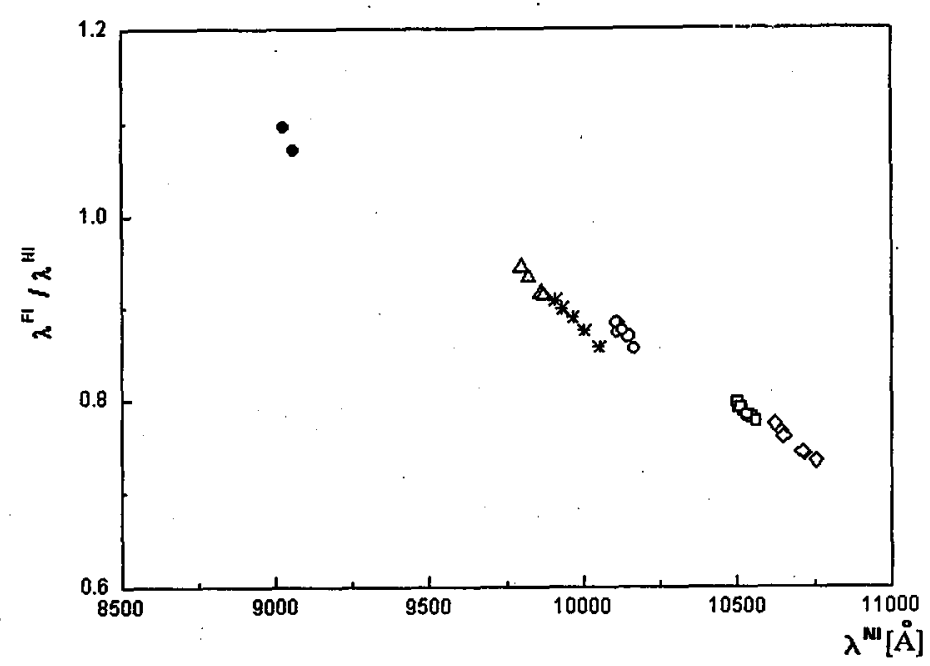

Fig. 6. The same as in Fig. 4 for $3 p-3 d$ transitions.

In Fig. 6 the corresponding wavelength ratios are plotted against the wavelength of the NI transition. A distinct regular trend is observed not only within but also amongst different multiplets.

\section{Conclusions}

In this paper absolute line strengths, recently determined experimentally by applying the emission spectroscopy method, are compared with the semi-empirical data of Kurucz and Peytremann, the results obtained by the Coulomb approximation method and with data resulting from the perturbation theory. The experimental absolute scales are based on lifetimes of excited levels of NI, FI and NeII taken from the literature. In the case of spectra NI and FI the absolute scales based on lifetime values agree satisfactorily with those resulting from Coulomb approximation. Larger discrepancies are encountered when the data for the isoelectronic emitters FI and NeII are analysed. In the average the CA method yields a significantly larger NeII / FI line strength ratio than those evaluated from experimental data based on lifetime values. In that case, however, satisfactory agreement is found between experimental data and those resulting from the perturbation theory. In spite of a rather regular trend of the absolute scale of respective multiplet strengths, the strengths for individual components exhibit in many cases very large departures from the LS coupling scheme, predominantly for spectral lines of the $3 p-3 d$ transition array. The overall consistence of experimental line strength values with the comprehensive semi-empirical data [22] is much better than those resulting from the LS coupling scheme.

\section{References}

[1] D. Luo, A.K. Pradhan, J. Phys. B 22, 3377 (1989).

[2] W. Cunto, C. Mendoza, Rev. Mex. Astron. Astrof. 23, 107 (1992). 
[3] H. Nussbaumer, P.J. Storey, Astron. Astrophys. 140, 383 (1984).

[4] A. Hibbert, E. Biemont, M. Godefroid, N. Vaeck, Astron. Astrophys. Suppl. Ser. 88, 505 (1991).

[5] K.L. Bell, C.A. Ramsbottom, A. Hibbert, J. Phys. B 25, 1735 (1992).

[6] A. Hibbert, E. Biemont, M. Godefroid, N. Vaeck, Astron. Astrophys. Suppl. Ser. 99, 179 (1993).

[7] K.L. Bell, A. Hibbert, R.P. Stafford, Phys. Scr. 52, 240 (1995).

[8] A.W. Weiss, to be published.

[9] C. Lavin, I. Martin, J. Quant. Spectrosc. Radiat. Transf. 50, 611 (1993).

[10] C. Lavin, I. Martin, J. Quant. Spectrosc. Radiat. Transf. 52, 21 (1994).

[11] A.M. Velasco, C. Lavin, I. Martin, J. Quant. Spectrosc. Radiat. Transf. 57, 509 (1993).

[12] J. Musielok, W.L. Wiese, G. Veres, Phys. Rev. A 31, 3588 (1995).

[13] J. Musielok, J.M. Bridges, S. Djurović, W.L. Wiese, Phys. Rev. A 53, 3122 (1996).

[14] G. Veres, W.L. Wiese, Phys. Rev. A 54, 1999 (1996).

[15] J. Musielok, G. Veres, W.L. Wiese, J. Quant. Spectrosc. Radiat. Transf. 57, 395 (1997).

[16] J. Musielok, E. Pawelec, U. Griesmann, W.L. Wiese, to be published.

[17] U. Griesmann, J. Musielok, W.L. Wiese, J. Opt. Soc. Am. B 14, 2204 (1997).

[18] H.G. Kuhn, Atomic Spectra, Academic Press, New York 1962.

[19] D.R. Bates, A. Damgaard, Philos. Trans. R. Soc. Lond. Math. Phys. Sci. A 242, 101 (1949).

[20] W.L. Wiese, A.W. Weiss; Phys. Rev. 175, 50 (1968).

[21] W.L. Wiese, in: Progrr.ss in Atomic Spectroscopy, Part B, Eds. W. Hanle, H. Kleinpoppen, Plenum Press, New York 1979, p. 1101.

[22] R.L. Kurucz, E. Peytremann, Smiths. Astrophys. Obs. Rep. 362 (1975).

[23] S. Bashkin, J.O. Stoner Jr., Atomic Energy Levels and Grotrian Diagrams, North Holland Publ. Co., Amsterdam 1975. 